\title{
TRATAMENTO NÃO FARMACOLÓGICO PARA SÍNDROME DOS OVÁRIOS POLICÍSTICOS
}

\section{CATEGORIA B: CLÍNICO. \\ CENTRO UNIVERSITÁRIO SÃO CAMILO}

PENIDO, F C B N ${ }^{1}$ - Endereço Rua Francisco Bondança, número 150- Telefone: (11) 99921-2360- Email: fernandabragapenido@ gmail.com.

\author{
Maia, L O ${ }^{1}$ \\ Furquim, $\mathbf{I}^{1}$ \\ Pereira, $\mathbf{M} \mathbf{M}^{2}$ \\ Vilarino, $\mathbf{F}^{2}$
}

1. Discente do Centro Universitário São Camilo

2. Docente do Centro Universitário São Camilo 


\title{
TRATAMENTO NÃO FARMACOLÓGICO PARA SÍNDROME DOS OVÁRIOS POLICÍSTICOS
}

\author{
CATEGORIA B: Clínico.
}

DESCRITORES: "Polycistic ovary syndrome"; "non-pharmacological treatment"; "PCOS" 


\title{
Tratamento não farmacológico da Síndrome dos Ovários Policísticos
}

AUTORES: Penido FC; Maia, LO; Souza, IF; ORIENTADOR: Vilarino, FL; Pereira, MM;

\author{
INSTITUIÇÃO: Centro Universitário São Camilo
}

INTRODUÇÃO: A síndrome dos ovários policísticos (SOP) é um distúrbio endócrino metabólico. Suas manifestações clínicas podem incluir irregularidades menstruais, sinais de hiperandrogenismo e obesidade. Contraceptivos orais e agentes sensibilizadores de insulina são a primeira linha de tratamento, entretanto, devido os efeitos colaterais, desencadeiam, baixa adesão frequentemente. Nesse sentido, surgem novas terapias alternativas no tratamento da SOP, que pretendem ter resultados iguais ou melhores em comparação às terapias tradicionais utilizadas.

OBJETIVO: Avaliar quais são e a eficácia dos tratamentos não farmacológicos da SOP.

METODOLOGIA: Utilizou-se a base de dados PubMed, foram encontrados 1069 artigos com o descritor "polycystic ovary syndrome AND non-pharmacologicol tratament" e 732 artigos com "PCOS AND non-pharmacologicol tratament". Após aplicação dos critérios de exclusão: Metanálises, artigos de revisão, relatos de caso e artigos com temas não compatíveis com o trabalho, foram selecionados 10 artigos publicados entre 2014 e 2018.

RESULTADOS: A Medicina Tradicional Chinesa (MTC) destacou-se entre os tratamentos não-farmacológicos. Estudos com ingestão diária, via oral de Trigonella (planta), obtiveram em $36 \%$ a regressão completa do cisto, retorno menstrual regular e aumento de LH e FSH. Outro estudo de 3 ciclos menstruais com MTC mostrou redução na reação inflamatória das pacientes. Houve melhora do IMC a partir de Eletroacupuntura maior do que no grupo que usou Diane-35 e há também estudos que sugeriram possibilidade da Cryptotanshinone (erva chinesa) normalizar a secreção de testosterona, progesterona e E2 em ratos com resistência à insulina ovariana induzida por dexametasona. Contudo, apenas um estudo comparou a EA com uma associação tradicionalmente utilizada no tratamento da SOP: Acetato de ciproterona e etinilestradiol. Neste, a taxa efetiva de melhora da SOP foi $2,7 \%$ maior nas pacientes que utilizaram o medicamento.

CONCLUSÃO: A literatura mostrou potencial da prática e dos medicamentos pertencentes à Medicina Tradicional Chinesa para o tratamento não farmacológico da SOP. Contudo, ainda faltam estudos que comparem essa abordagem aos principais tratamentos farmacológicos dessa síndrome. Além disso, uma vez que a maioria dos estudos foram realizados com grupos específicos, os resultados não podem ser necessariamente extrapolados à população brasileira.

DESCRITORES: polycystic ovary syndrome, PCOS. 


\section{INTRODUÇÃO}

A síndrome dos ovários policísticos (SOP) é um distúrbio endócrino metabólico. Suas manifestações clínicas podem incluir irregularidades menstruais, sinais de excesso de andrógenos e obesidade. Ela acomete cerca de 6-18\% das mulheres em idade fértil. ${ }^{1,2}$

A patogênese da SOP é complexa e a definição da doença se dá por 2 dos 3 critérios de Rotterdam, descritos abaixo:

1. Oligomenorréia (menstruação com intervalo maior que 35 dias) e/ou anovulação

2. Sinais clínicos e/ou bioquímicos de hiperandrogenismo, excluindo outras etiologias de hiperandrogenismo como hiperplasia adrenal congênita, tumores secretores de androgênios e síndrome de Cushing.

3. Ovários policísticos caracterizados pelo exame ultrassonográfico padronizado, ou seja, presença de pelo menos um dos seguintes achados: 12 ou mais folículos medindo entre 2-9 mm de diâmetro ou volume ovariano aumentado $\left(>10 \mathrm{~cm}^{3}\right)$. Caso se constate a presença de um folículo dominante (>10 mm) ou de corpo lúteo, o ultra-som (US) deverá ser repetido no próximo ciclo. 3,4

Contraceptivos orais e agentes sensibilizadores de insulina são considerados manejo de atenção primária à Síndrome dos Ovários Policísticos, mas efeitos colaterais intoleráveis, baixa adesão e potencial aumento de risco cardiovascular e metabólico destacam a importância dos tratamentos não farmacológicos para esta síndrome.

Nesse sentido surgem novas terapias alternativas para o tratamento da SOP, que pretendem ter resultados iguais ou melhores em comparação aos contraceptivos orais e os agentes sensibilizadores de insulina, mas com menos efeitos colaterais. Assim, esse estudo tem como objetivo avaliar, por meio da literatura, quais são os tratamentos não farmacológicos para SOP e a sua eficácia.

\section{METODOLOGIA}

Para esta Revisão Sistemática foi utilizada a base de dados PubMed. Foram encontrados 1069 artigos com o descritor "polycystic ovary syndrome AND non-pharmacological treatment" e 732 artigos com "PCOS AND non-pharmacological treatment". Foram excluídos metanálises, artigos de revisão, relatos de caso e artigos com temas não compatíveis com o trabalho. Foram incluídos 10 artigos publicados entre $\mathrm{o}$ ano de 2014 e 2018.

\section{RESULTADOS:}

\begin{tabular}{|c|c|c|c|c|c|}
\hline Nome & Ano & $\begin{array}{l}\text { Tipo de } \\
\text { Estudo }\end{array}$ & Amostra & Intervenção & Resultados \\
\hline $\begin{array}{l}\text { Efficacy comparison } \\
\text { between } \\
\text { electroacupuncture } \\
\text { and dyne- } 35 \text { in } \\
\text { treatment of } \\
\text { polycystic ovary } \\
\text { syndrome } 5\end{array}$ & 2014 & $\begin{array}{l}\text { Ensaio } \\
\text { Clínico } \\
\text { Randomiza } \\
\text { do }\end{array}$ & $\begin{array}{l}65 \\
\text { mulheres } \\
\text { com } \\
\text { SOP }\end{array}$ & $\begin{array}{l}\text { Estimulação } \\
\text { elétrica (EA) } \\
\text { nos pontos } \\
\text { de } \\
\text { acupuntura }\end{array}$ & $\begin{array}{l}\text { A taxa efetiva: } 90,6 \% \\
\text { no grupo } E A \text { e } 93,3 \% \\
\text { no grupo de dyne- } 35 \text {. } \\
\text { Níveis de LH e LH / } \\
\text { FSH foram reduzidos } \\
\text { nos dois grupos } \\
\text { (todos } \mathrm{P}<0,01 \text { ). }\end{array}$ \\
\hline
\end{tabular}




\begin{tabular}{|c|c|c|c|c|c|}
\hline & & & & & $\begin{array}{l}\text { A diferença na massa } \\
\text { corporal e IMC: o grupo EA } \\
\text { foi mais significativa }\end{array}$ \\
\hline $\begin{array}{lr}\text { Preliminary } & \text { evidence } \\
\text { that } & \text { cinnamon } \\
\text { improves } & \text { menstrual } \\
\text { cyclicity in women with } \\
\text { polycystic } & \text { ovary } \\
\text { syndrome: } & \text { a } \\
\text { randomized } & \text { controlled } \\
\text { trial }^{6} & \end{array}$ & 2014 & $\begin{array}{l}\text { Ensaio } \\
\text { Clínico } \\
\text { Randomiza } \\
\text { do }\end{array}$ & $\begin{array}{l}45 \\
\text { mulheres } \\
\text { com SOP }\end{array}$ & $\begin{array}{l}\text { Suplement } \\
0 \quad \text { de } \\
\text { canela }(1,5 \\
\text { g/dia) }\end{array}$ & $\begin{array}{ll}\text { A ciclicidade menstrual } \\
\text { melhorou (+ } 0,23 \text { ciclos / } \\
\text { mês) Medidas de } \\
\text { resistência à insulina ou } \\
\text { níveis séricos } & \text { de } \\
\text { andrógeno não se } \\
\text { alteraram. }\end{array}$ \\
\hline $\begin{array}{l}\text { Oriental medicine } \\
\text { Kyung-Ok-Ko } \\
\text { prevents and } \\
\text { alleviates } \\
\text { dehydroepiandroster } \\
\text { one-induced } \\
\text { polycystic ovarian } \\
\text { syndrome in rats } 7\end{array}$ & $\begin{array}{l}201 \\
4\end{array}$ & $\begin{array}{l}\text { Estudo } \\
\text { prospectiv } \\
0\end{array}$ & $\begin{array}{l}56 \text { ratos } \\
\text { fêmeas }\end{array}$ & $\begin{array}{l}\text { KOK (0,5, } \\
1,0 \text { e } 2,0 \mathrm{~g} / \\
\mathrm{kg} / \text { dia, VO) } \\
\text { Composição } \\
\text { da KOK: } \\
\text { Rehmannia } \\
\text { glutinosa } \\
\text { Liboschitz } \\
\text { var. } \\
\text { Purpurae } \\
\text { Makino (32,0 } \\
\text { g), pó de } \\
\text { frutos secos } \\
\text { de Lycium } \\
\text { chinense } \\
\text { Miller (0,9 } \\
\text { g), pó de } \\
\text { resina de } \\
\text { Aquillaria } \\
\text { agallocha } \\
\text { Roxburgh } \\
\text { (0,1 g), pó de } \\
\text { córtex de } \\
\text { Poria cocos } \\
\text { Wolf ( } 8,0 \text { g), } \\
\text { pó de raiz } \\
\text { seca de } \\
\text { Panax } \\
\text { Ginseng CA } \\
\text { Meyer ( } 2,8 \\
\text { g), mel ( } 38,5 \\
\text { g de mel } \\
\text { natural) e } \\
\text { xarope } \\
\text { simples (17,7 } \\
\text { g de soluto }\end{array}$ & $\begin{array}{l}\text { Os pesos ovarianos: } \\
\text { no grupo DHEA } \\
\text { (dehydroepiandroster } \\
\text { one) foram } 26,7 \% \\
\text { maior do que no } \\
\text { grupo normal. Houve } \\
\text { uma diminuição de } \\
30,8 \% \text { com KOK. } \\
\text { O número de cistos } \\
\text { foliculares nos } \\
\text { ovários no grupo } \\
\text { DHEA foi de } 6,2 \pm \\
\text { 0,7; e no grupo } \\
\text { DHEA + KOK foi de } \\
3,4 \pm 0,7 . \\
\text { O aumento do nível } \\
\text { sérico de } \\
\text { progesterona no } \\
\text { grupo DHEA (em } 3,6 \\
\text { vezes) foi } \\
\text { discretamente } \\
\text { diminuído no grupo } \\
\text { DHEA + KOK (em } \\
27,6 \% \text { ). } \\
\text { Efeito do KOK após a } \\
\text { administração de } \\
\text { DHEA: } \\
\text { KOK inibiu o } \\
\text { aumento do peso } \\
\text { corporal (em } 4,8 \% \text { ), } \\
\text { aumentou os níveis } \\
\text { séricos de estradiol } \\
\text { (em } 61,6 \% \text { ), } \\
\text { aumentou o número } \\
\text { ( } 84,6 \% \text { ) e o tamanho } \\
\text { dos cistos foliculares. }\end{array}$ \\
\hline
\end{tabular}




\begin{tabular}{|c|c|c|c|c|c|}
\hline & & & & $\begin{array}{l}\text { de sacarose } \\
\text { a } 85 \% \text { ) }\end{array}$ & $\begin{array}{l}\text { A administração da KOK } \\
\text { mostrou efeitos positivos na } \\
\text { diminuição de estado } \\
\text { inflamatório induzido pela } \\
\text { SOP. }\end{array}$ \\
\hline $\begin{array}{l}\text { Effect of Bushen } \\
\text { Huatan Recipe on } \\
\text { the Akt signal } \\
\text { pathway in } \\
\text { polycystic ovarian } \\
\text { syndrome model rats } \\
\text { with insulin } \\
\text { resistance: an } \\
\text { experimental } \\
\text { research }\end{array}$ & $\begin{array}{l}201 \\
4\end{array}$ & $\begin{array}{l}\text { Estudo } \\
\text { prospectiv } \\
0\end{array}$ & $\begin{array}{l}50 \text { ratos } \\
\text { com } \\
\text { SOP }\end{array}$ & $\begin{array}{l}\text { BHR de } \\
\text { baixa dose } \\
(5,406 \mathrm{~g} / \\
\mathrm{kg}), \text { grupo } \\
\text { BHR de dose } \\
\text { média } \\
(10,812 \mathrm{~g} / \\
\mathrm{kg}) \text { e grupo } \\
\text { BHR de alta } \\
\text { dose }(21,624 \\
\mathrm{g} / \mathrm{kg})\end{array}$ & $\begin{array}{l}\text { No grupo modelo: } \\
\text { HOMA-IR e a } \\
\text { expressão de mRNA } \\
\text { do PPAR-gama } \\
\text { aumentaram } \\
\text { significativamente, a } \\
\text { expressão do mRNA } \\
\text { da expressão da } \\
\text { proteína GSK-3beta, } \\
\text { GLUT-4 e IRS-1 de } \\
\text { Akt e p-Akt diminuiu } \\
\text { (P <0,05). } \\
\text { Comparado com o } \\
\text { grupo modelo, } \\
\text { HOMA-IR diminuiu } \\
\text { significativamente, a } \\
\text { expressão de mRNA } \\
\text { da GSK-3beta, } \\
\text { GLUT-4, IRS-1 e } \\
\text { proteína Akt } \\
\text { aumentou } \\
\text { significativamente no } \\
\text { grupo BHR alta (P } \\
<0,05, P<0,01) \text {, } \\
\text { média e baixa dose. }\end{array}$ \\
\hline $\begin{array}{l}\text { Cryptotanshinone } \\
\text { reverses ovarian } \\
\text { insulin resistance in } \\
\text { mice through } \\
\text { activation of insulin } \\
\text { signaling and the } \\
\text { regulation of } \\
\text { glucose transporters } \\
\text { and hormone } \\
\text { synthesizing } \\
\text { enzymes. }\end{array}$ & $\begin{array}{l}201 \\
4\end{array}$ & $\begin{array}{l}\text { Estudo } \\
\text { prospectiv } \\
0\end{array}$ & 60 ratos & $\begin{array}{l}\text { A resistência } \\
\text { à insulina } \\
\text { ovariana foi } \\
\text { induzida por } \\
\text { dexametaso } \\
n \\
\text { a (DEX). O } \\
\text { tratamento } \\
\text { foi com CRY }\end{array}$ & $\begin{array}{l}\text { Houve superovulação } \\
\text { no grupo com uso de } \\
\text { dexametasona e } \\
\text { ovulação próxima do } \\
\text { controle nos grupos } \\
\text { em que foi dado CRY } \\
\text { e resultados } \\
\text { semelhantes in vitro. } \\
\text { A criptotansinona } \\
\text { atenuou a inibição da } \\
\text { DEX na AKT2 e } \\
\text { suprimiu a regulação } \\
\text { positiva da expressão } \\
\text { da CYP11 e CYP17 } \\
\text { pela DEX. }\end{array}$ \\
\hline
\end{tabular}




\begin{tabular}{|c|c|c|c|c|c|}
\hline $\begin{array}{l}\text { Serum } \\
\text { metabolomics study } \\
\text { of Traditional } \\
\text { Chinese medicine } \\
\text { formula intervention } \\
\text { to polycystic ovary } \\
\text { syndrome }{ }^{10}\end{array}$ & 2015 & $\begin{array}{l}\text { Estudo } \\
\text { prospectiv } \\
0\end{array}$ & $\begin{array}{l}30 \\
\text { mulheres } \\
\text { com } \\
\text { SOP }\end{array}$ & $\begin{array}{l}3 \text { ciclos } \\
\text { menstruais } \\
\text { com } \\
\text { Medicina } \\
\text { Tradicional } \\
\text { Chinesa } \\
\text { (TCM), } \\
\text { Bushen } \\
\text { Huatan } \\
\text { Formula } \\
\text { (BHF) }\end{array}$ & $\begin{array}{l}\text { Os níveis séricos de } \\
\text { glicerofosforiletanola } \\
\text { mina (GPEA), } \\
\text { creatina, creatinina } \\
\text { diminuiu nos grupos } \\
\text { Normo Insulinico } \\
\text { (NI) e High Insulinico } \\
\text { (HI). Combinado com } \\
\text { dados clínicos } \\
\text { bioquímicos, a BHF } \\
\text { mostrou-se eficaz } \\
\text { para a SOP, } \\
\text { reduzindo a reação } \\
\text { inflamatória e os } \\
\text { níveis de estresse. }\end{array}$ \\
\hline $\begin{array}{l}\text { Efficacy of a Novel } \\
\text { Fenugreek Seed } \\
\text { Extract (Trigonella } \\
\text { foenum-graecum, } \\
\text { Furocyst) in } \\
\text { Polycystic Ovary } \\
\text { Syndrome (PCOS) I I }\end{array}$ & $\begin{array}{l}201 \\
5\end{array}$ & $\begin{array}{l}\text { Ensaio } \\
\text { Clínico } \\
\text { Randomiza } \\
\text { do }\end{array}$ & $\begin{array}{l}50 \\
\text { mulheres } \\
\text { em } \\
\text { pré-men } \\
\text { opausa } \\
\text { com } \\
\text { SOP }\end{array}$ & $\begin{array}{l}\text { Extrato de } \\
\text { sementes de } \\
\text { Trigonella } \\
\text { foenum-grae } \\
\text { cum }\end{array}$ & $\begin{array}{l}46 \% \text { das mulheres } \\
\text { obtiveram redução no } \\
\text { tamanho do cisto, } \\
36 \% \text { apresentaram } \\
\text { dissolução completa } \\
\text { do cisto. } 71 \% \text { dos } \\
\text { indivíduos relataram } \\
\text { o retorno do ciclo } \\
\text { menstrual regular e } \\
12 \% \text { dos indivíduos } \\
\text { subsequentemente } \\
\text { engravidaram. }\end{array}$ \\
\hline $\begin{array}{l}\text { Potent therapeutic } \\
\text { effects of shouwu } \\
\text { jiangqi decoction } \\
\text { (SJD) on polycystic } \\
\text { ovary syndrome } \\
\text { with insulin } \\
\text { resistance in rats }{ }^{1<}\end{array}$ & $\begin{array}{l}201 \\
5\end{array}$ & $\begin{array}{l}\text { Estudo } \\
\text { prospectiv } \\
0\end{array}$ & 51 ratos & $\begin{array}{l}\text { SJD: Radix } \\
\text { Polygoni } \\
\text { Multiflori, } \\
\text { Bombyx } \\
\text { Batryticatus, } \\
\text { Radix } \\
\text { Astragali, } \\
\text { Rhizoma } \\
\text { Dioscoreae, } \\
\text { Euonymus } \\
\text { alatus, the } \\
\text { Rhizoma } \\
\text { Cyperi }\end{array}$ & $\begin{array}{l}\text { O nível de FSH } \\
\text { diminuiu } \\
\text { significativamente no } \\
\text { grupo modelo em } \\
\text { comparação com o } \\
\text { controle normal ( } P \\
<0,01 \text { ), e altas doses } \\
\text { de SJD e } \\
\text { Dimethylbiguanide } \\
\text { (DMBG) podem } \\
\text { aumentar } \\
\text { significativamente o } \\
\text { nível de FSH (P } \\
<0,01 \text { ). } \\
\text { DMBG pode diminuir } \\
\text { significativamente o } \\
\text { nível de LH (P } \\
<0,01 \text { ). Altas doses de } \\
\text { DMJ e DMBG podem } \\
\text { diminuir } \\
\text { significativamente o } \\
\text { nível de testosterona }\end{array}$ \\
\hline
\end{tabular}




\begin{tabular}{|c|c|c|c|c|c|}
\hline & & & & & $\begin{array}{l}(P<0,01) \text {. A } \\
\text { expressão de IRS-1, bem } \\
\text { como PI3Kp85a foram } \\
\text { significativamente } \\
\text { diminuídos no grupo modelo } \\
\text { em comparação com o } \\
\text { grupo controle normal, tanto } \\
\text { no nível mRNA }(P<0,001) \text { e } \\
\text { proteína }(P<0,01), \text { e ambos } \\
\text { alta dose SJD e DMBG } \\
\text { pode melhorar a expressão } \\
\text { de IRS-1 e PI3K ( } P \\
<0,05) \text {. }\end{array}$ \\
\hline $\begin{array}{l}\text { Infertility in polycystic } \\
\text { ovary syndrome } \\
\text { treated } \\
\text { acupuncture andh } \\
\text { clomiphene: and } \\
\text { randomized } \\
\text { controlled trial }\end{array}$ & $\begin{array}{l}201 \\
5\end{array}$ & $\begin{array}{l}\text { Ensaio } \\
\text { Clínico } \\
\text { Randomiza } \\
\text { do }\end{array}$ & $\begin{array}{l}120 \\
\text { mulheres }\end{array}$ & $\begin{array}{l}\text { Um grupo } \\
\text { clomifeno } \\
\text { (grupo A), } \\
\text { um } \\
\text { acupuntur } \\
\text { a- } \\
\text { moxabustã } \\
\text { o } \\
+ \text { grupo de } \\
\text { medicina } \\
\text { chinesa } \\
\text { (grupo B) e } \\
\text { um } \\
\text { clomifeno } \\
+ \\
\text { acupuntur } \\
\text { a- } \\
\text { moxabustã } \\
\text { o } \\
+ \text { grupo } \\
\text { de } \\
\text { medicina } \\
\text { chinesa } \\
\text { (grupo C). }\end{array}$ & $\begin{array}{l}\text { Escore de muco cervical de } \\
\text { HCG dia, espessura do } \\
\text { endométrio e morfologia } \\
\text { endometrial (taxa de tipo A): } \\
\text { os resultados no grupo C } \\
\text { foram melhores que os do } \\
\text { grupo A; os resultados no } \\
\text { grupo B foram melhores que } \\
\text { os do grupo A. A diferença } \\
\text { na espessura endometrial } \\
\text { não foi significativa na } \\
\text { comparação entre o grupo C } \\
\text { e o grupo B ( } P>0,05 \text { ). O } \\
\text { escore de muco cervical e } \\
\text { morfologia endometrial } \\
\text { (taxa de tipo A) no grupo } C \\
\text { foram melhores do que } \\
\text { aqueles no grupo B (ambos } \\
\mathrm{P}<0,05) \text {. } \\
\text { As taxas de ciclo ovulatório } \\
\text { no grupo } A \text { e no grupo } \mathrm{C} \\
\text { foram maiores que no grupo } \\
\mathrm{B} \text { (ambos } \mathrm{P}<0,05 \text { ), a taxa de } \\
\text { gravidez no grupo } \mathrm{C} \text { foi } \\
\text { maior que }\end{array}$ \\
\hline
\end{tabular}




\begin{tabular}{|c|c|c|c|c|c|}
\hline & & & & & $\begin{array}{l}\text { nos outros grupos (ambos } P \\
<0,05) \text {, e a taxa de aborto } \\
\text { precoce no grupo } C \text { foi } \\
\text { menor do que no grupo } A \text { e } \\
\text { no grupo } B \text { (ambos } P<0,01 \text { ) }\end{array}$ \\
\hline $\begin{array}{l}\text { Standardised versus } \\
\text { individualised } \\
\text { multiherb Chinese } \\
\text { herbal medicine for } \\
\text { oligomenorrhoea } \\
\text { and amenorrhoea in } \\
\text { polycystic ovary } \\
\text { syndrome: a } \\
\text { randomised } \\
\text { feasibility and pilot } \\
\text { study in the UK. }{ }^{14}\end{array}$ & $\begin{array}{l}201 \\
7\end{array}$ & $\begin{array}{l}\text { Ensaio } \\
\text { Clínico } \\
\text { Randomiza } \\
\text { do }\end{array}$ & $\begin{array}{l}40 \\
\text { mulheres } \\
\text { com } \\
\text { SOP }\end{array}$ & $\begin{array}{l}6 \text { meses de } \\
\text { CHM } \\
\text { padronizado } \\
\text { ou CHM } \\
\text { individualiza } \\
\text { do, } 16 \mathrm{~g} \\
\text { diários } \\
\text { tomados } \\
\text { oralmente } \\
\text { como chá }\end{array}$ & $\begin{array}{l}\text { Melhoras estatísticas } \\
\text { significativas nas } \\
\text { taxas menstruais } \\
\text { foram encontradas em } \\
6 \text { meses dentro do } \\
\text { grupo CHM } \\
\text { padronizado } \\
\text { (diferença média } \\
\text { (MD) } 0,18 \pm 0,06, \text { IC } \\
95 \% 0,06-0,29 ; p= \\
0,0027 \text { ) e } \\
\text { individualizado CHM } \\
\text { (MD 0,27 } \pm 0,06, \text { IC } \\
95 \% 0,15 \text { a } 0,39 ; p \\
<0,001 \text { ). } \\
\text { Nenhuma melhora foi } \\
\text { observada para o IMC } \\
\text { nem para o peso. As } \\
\text { melhorias nos escores } \\
\text { de hirsutismo } \\
\text { encontrados dentro do } \\
\text { grupo para ambos os } \\
\text { grupos não foram } \\
\text { estatisticamente } \\
\text { significantes entre os } \\
\text { grupos ( } p=0,09 \text { ). }\end{array}$ \\
\hline
\end{tabular}

\section{DISCUSSÃO:}

A etiologia da SOP não está completamente elucidada. No entanto, várias hipóteses são listadas, como: Alterações na liberação pulsátil de gonadotrofinas $(\mathrm{GnRH})$; na liberação hipofisária dos hormônios luteinizante (LH) e folículo-estimulante (FSH); nas funções ovariana e supra-renal e a resistência insulínica. Assim, as concentrações séricas de LH são freqüentemente elevadas nestes pacientes. Tanto o nível absoluto de LH circulante quanto a sua relação com os níveis de FSH são significativamente elevados em mulheres com SOP em comparação aos controles. ${ }^{15,16}$

Estima-se que, no mundo, 105 milhões de mulheres entre 15 e 49 anos de idade (sendo 4 milhões americanas) apresentam SOP, a qual é responsável por 72 a $82 \%$ das causas de hiperandrogenismo. ${ }^{17}$

A SOP provoca alterações relacionadas ao hiperandrogenismo, como hirsutismo e aparecimento de acne e também alterações relacionada à resistência insulínica. A resistência insulínica confere diminuição da sensibilidade dos tecidos à ação do hormônio, o que implica no mau 
funcionamento do metabolismo, como diminuição da função das células beta pancreáticas e intolerância à glicose. Além disso, evidências demonstraram a ação da insulina e dos fatores insulinóides de crescimento (IGF I e II) sobre o desenvolvimento do folículo ovariano e sobre a estimulação da síntese de androgênios nas células da teca interna in vitro. A hiperinsulinemia também está relacionada com a redução da síntese de SHBG no fígado e das proteínas carreadoras dos fatores insulinóides. ${ }^{18,19}$

Abaixo, descrevem-se as técnicas para avaliar a presença de resistência insulínica:

- índice HOMA, medida da insulina em $\mathrm{mUI} / \mathrm{l}$ x glicemia em mmol/dl / 22,5;

- teste de tolerância oral à glicose (TTOG), que consiste na administração de $75 \mathrm{~g}$ de glicose e, a seguir, faz-se a determinação de glicemia e insulina nos tempos 0, 30, 60 e 120 minutos;

- teste de tolerância à glicose simplificado, no qual se faz as dosagens nos tempos 0 e 120 minutos;

- relação entre glicemia e insulina $(G / l)$ de jejum, cujo valor considerado normal é menor que 4,5;

- teste QUICKI (quantitative sensitivity check index), cujo resultado é obtido com a seguinte fórmula matemática: QUICKI = 1 / [Log insulina de jejum insulin + Log glicemia de jejum]. 20,21

Para estabelecer o diagnóstico de SOP, é importante excluir outros distúrbios com uma apresentação clínica semelhante, como hiperplasia adrenal congênita, síndrome de Cushing e tumores secretores de andrógenos. Apesar da exclusão rotineira da disfunção tireoidiana em pacientes considerados hiperandrogênicos ser questionada, como o rastreamento de distúrbios da tireoide, pode ser aconselhável em todas as pacientes hiperandrogênicas em idade reprodutiva realizar a medição do TSH. A investigação inicial em mulheres que apresentam oligomenorreia / anovulação também pode incluir a avaliação dos níveis séricos de FSH e E2 para excluir hipogonadismo hipogonadotrófico ou falência ovariana prematura caracterizada por baixas concentrações de E2 e FSH. 22,23,24

Além da resistência insulínica, a SOP está relacionada com a síndrome metabólica. A síndrome metabólica ocorre em aproximadamente $43 \%$ das pacientes com SOP, elevando em até sete vezes o risco de doença cardiovascular nestas pacientes. A SM é caracterizada por no mínimo 3 das características apresentadas a seguir 25 :

\begin{tabular}{|lc|}
\hline \multicolumn{2}{|c|}{ Quadro 1 - Componentes da síndrome metabólica segundo o } \\
NCEP-ATP III
\end{tabular}


O tratamento da SOP tem como alvo diminuir a produção de andrógenos, restaurar a função ovulatória e regular os ciclos menstruais. Além disso, o tratamento medicamentoso da SOP também deve visar reduzir o risco da Síndrome Metabólica. Os principais medicamentos utilizados são contraceptivos orais e agentes sensibilizadores de insulina. Os efeitos colaterais intoleráveis, baixa adesão e potencial aumento de risco cardiovascular e metabólico instigam a busca dos tratamentos não farmacológicos para esta síndrome.

A acupuntura, ciência praticada na medicina tradicional chinesa, é conhecida há mais de dois milênios e usada no tratamento de muitas doenças principalmente para o alívio de estímulos dolorosos. Desde 1960 a acupuntura com eletroestimulação (EA) é usada para curar desordens anovulatórias. De acordo com a literatura, a taxa de indução de EA na ovulação aumentou de $50 \%$ inicialmente para $80 \%$ atualmente. Outros autores chineses também relataram sucesso no tratamento de pacientes com esterilidade e mulheres com contração do útero anormal. Mesmo que as pesquisas demonstrem que a acupuntura pode ser um método curativo eficaz das doenças de algumas mulheres, os mecanismos de ação dessa prática são desconhecidos.

De acordo com CHEN, B. Y. a observação clínica mostrou que Eletroacupuntura (EA) com os pontos de acupuntura eficazes poderia curar alguns pacientes anovulatórios em uma taxa muito eficaz e os resultados experimentais sugeriram que EA poderia regular a disfunção do eixo hipotálamo-hipófise-ovário de várias maneiras, o que poderia influenciar alguma expressão gênica do cérebro, assim, normalizando secreção de alguns hormônios, como GnRH, LH e E2. ${ }^{27}$

Além disso, os estudos que avaliaram os tratamentos não farmacológicos derivados de ervas chinesas ou práticas da Medicina Tradicional Chinesa mostraram melhora na resistência à insulina do grupo de intervenção em relação ao grupo com SOP, melhora do IMC, redução de peso e do aspecto policístico do ovário.

Nenhum estudo revelou qualquer efeito adverso com a intervenção. Apenas um estudo comparou a intervenção (Estimulação elétrica nos pontos de acupuntura) com um dos principais medicamentos utilizado no tratamento da SOP, Diane-35. Neste, a taxa efetiva de tratamento da SOP foi de $2,7 \%$ de diferença entre pacientes que utilizaram Diane-35 e as que utilizaram EA, porém a diferença na massa corporal e no IMC foi mais significativa no grupo em que foi administrada EA.

\section{CONCLUSÃO:}

A literatura mostrou o potencial de certas ervas chinesas, bem como a prática da Medicina Tradicional Chinesa, como acupuntura para o tratamento não-farmacológico da SOP através de mecanismos como melhora nos níveis hormonais de estrogênio, progesterona e testosterona, melhora na resistência à insulina e diminuição de peso e IMC. Contudo ainda faltam estudos que comparem essa abordagem aos principais tratamentos para a SOP, como contraceptivos orais e agentes sensibilizadores de insulina.

Além disso, uma vez que a maioria dos estudos foram realizados com grupos específicos, e os resultados não podem ser necessariamente extrapolados, a aplicabilidade dessas intervenções na população brasileira necessita de mais dados para embasar esta medida. 


\section{REFERÊNCIAS:}

1. Assunção M, Calvo RM, San Millán JL, et al. Um estudo prospectivo da prevalência da síndrome dos ovários policísticos em mulheres caucasianas não selecionadas da Espanha . J Clin Endocrinol Metab 2000 ; 85 : 2434 - 8 . http://dx.doi.org/10.1210/jcem.85.7.6682

2. Março de WA ,Moore VM ,Willson KJ , et al. A prevalência da síndrome dos ovários policísticos em uma amostra da comunidade avaliada sob critérios diagnósticos contrastantes . Hum Reprod 2010 ; 25 : 544 - 51 . doi: 10.1093 / humrep / dep399

3. Balen AH, Laven JSE, Dewailly D. Ultrasound assessment of the polycystic ovary: international consensus definition. Hum Reprod 2003; 9(6):505-14.

4. The Rotterdam ESHRE/ASRM-sponsored PCOS consensus workshop group. Revised 2003 consensus on diagnostic criteria and long-term health risks to polycystic ovary syndrome (PCOS). Hum Reprod 2004; 19(1):41-7.

5. Jin C, Wei L, Zhao J, Wu Z. [Efficacy comparison between electroacupuncture and dyne-35 in treatment of polycystic ovary syndrome]. Zhongguo Zhen Jiu = Chinese Acupuncture \& Moxibustion [serial on the Internet]. (2014, Dec), [cited July 24, 2018]; 34(12): 1174-1178.

6. Kort D, Lobo R. Preliminary evidence that cinnamon improves menstrual cyclicity in women with polycystic ovary syndrome: a randomized controlled trial. American Journal Of Obstetrics And Gynecology [serial on the Internet]. (2014, Nov), [cited July 24, 2018]; 211(5): 487.e1-6.

7. Jang M, Lee M, Lee J, Bae C, Kim S, Cho I, et al. Oriental medicine Kyung-Ok-Ko prevents and alleviates dehydroepiandrosterone-induced polycystic ovarian syndrome in rats. Plos One [serial on the Internet]. (2014, Feb 10), [cited July 24, 2018]; 9(2): e87623.

8. Hong Y, Wu F. [Effect of Bushen Huatan Recipe on the Akt signal pathway in polycystic ovarian syndrome model rats with insulin resistance: an experimental research]. Zhongguo Zhong Xi Yi Jie He Za Zhi Zhongguo Zhongxiyi Jiehe Zazhi = Chinese Journal Of Integrated Traditional And Western Medicine [serial on the Internet]. (2014, Feb), [cited July 24, 2018]; 34(2): 230-234.

9. Huang Y, Li W, Wang C, Wu X, Zheng J. Cryptotanshinone reverses ovarian insulin resistance in mice through activation of insulin signaling and the regulation of glucose transporters and hormone synthesizing enzymes. Fertility And Sterility [serial on the Internet]. (2014, Aug), [cited July 24, 2018]; 102(2): 589-596.e4. Available from: MEDLINE Complete.

10.Lu C, Zhao X, Li Y, Li Y, Yuan C, Xu G, et al. Serum metabolomics study of Traditional Chinese medicine formula intervention to polycystic ovary syndrome. Journal Of Pharmaceutical And Biomedical Analysis [serial on the Internet]. (2016, Feb 20), [cited July 24, 2018]; 120127-133

11. Swaroop A, Jaipuriar A, Gupta S, Bagchi M, Kumar P, Bagchi D, et al. Efficacy of a Novel Fenugreek Seed Extract (Trigonella foenum-graecum, Furocyst) in Polycystic Ovary Syndrome (PCOS). International Journal Of Medical Sciences [serial on the Internet]. (2015, Oct 3), [cited July 24, 2018]; 12(10): 825-831.

12. Wang L, Wang $\mathrm{X}, \mathrm{Yu} \mathrm{X}, \mathrm{Xu} \mathrm{W}$. Potent therapeutic effects of shouwu jiangqi decoction on polycystic ovary syndrome with insulin resistance in rats. Chinese Journal Of Integrative Medicine [serial on the Internet]. (2016, Feb), [cited July 24, 2018]; 22(2): 116-123. 
13. Jiang D, Zhang Y, Wu X, Wu S. [Infertility in polycystic ovary syndrome treated with acupuncture and clomiphene: a randomized controlled trial]. Zhongguo Zhen Jiu = Chinese Acupuncture \& Moxibustion [serial on the Internet]. (2015, Feb), [cited July 24, 2018]; 35(2): 114-118.

14. Lai L, Flower A, Prescott $P$, Wing $T$, Moore $M$, Lewith $G$. Standardised versus individualised multiherb Chinese herbal medicine for oligomenorrhoea and amenorrhoea in polycystic ovary syndrome: a randomised feasibility and pilot study in the UK. BMJ Open [serial on the Internet]. (2017, Feb 3), [cited July 24, 2018]; 7(2): e011709.

15. Fauser BC, Pache TD, Lamberts SW, Hop WC, de Jong FH, Dahl KD. Serum bioactive and immunoreactive LH and FSH levels in women with cycle abnormalities, with or without PCOD. J Clin Endocrinol Metab 1991;73:811-7.

16. Taylor AE, McCourt B, Martin K, Anderson EJ, Adams J, Schoebfeld D, et al. Determinants of abnormal gonadotropin secretion in clinically de $\square$ ned women with PCOS. J Clin Endocrinol Metab 1997;82:2248- 56.

17. Azziz R, Marin C, Hoq L, Badamgarav E, Song P. Health care-related economic burden of the polycystic ovary syndrome during the reproductive life span. The Journal Of Clinical Endocrinology And Metabolism [serial on the Internet]. (2005, Aug), [cited July 25, 2018]; 90(8): 4650-4658.

18. Teixeira Filho FL, Baracat EC, Lee TH, Suh CS, Matsui M, Chang RJ, Shimasaki $\mathrm{S}$, Erickson GF. Aberrant expression of growth differentiation factor-9 in oocytes of women with polycystic ovary syndrome. J Clin Endocrinol Metab. 2002;87(3):1337-44.

19. Giudice LC. Endometrium in PCOS: Implantation and predisposition to endocrine CA. Best Pract Res Clin Endocrinol Metab. 2006;20(2):235-44

20. Barber TM, Wass JA, McCarthy MI, Franks S. Metabolic characteristics of women with polycystic ovaries and oligo-amenorrhoea but normal androgen levels: implications for the management of polycystic ovary syndrome. Clin Endocrinol (Oxf). 2007;66(4):513-7.

21. de Paula Martins W, Santana LF, Nastri CO, Ferriani FA, de Sa MF, Dos Reis RM. Agreement among insulin sensitivity indexes on the diagnosis of insulin resistance in polycystic ovary syndrome and ovulatory women. Eur J Obstet Gynecol Reprod Biol. 2007 Jan 4; [Epub ahead of print]

22. Azziz R, Hincapie LA, Knochenhauer ES, Dewailly D, Fox L, Boots LR. Screening for 21-hydroxylase de $\square$ cient non-classic adrenal hyperplasia among hyperandrogenic women: a prospective study. Fertil Steril 1999;72:915-25.

23. ESHRE Capri Workshop Group. Anovulatory infertility. Hum Reprod 1995;10:1549-53

24. Rowe PJ, Comhaire FH, Hargreave TB. Female partner. In: World Health Organization manual for the standardized investigation and diagnosis of the infertile couple. Cambridge: Cambridge University Press, 2000:40-67. 18. Van Santbrink EJ, Hop WC, Fauser BC. Classi $\square$ cation of normogonadotropin infertility: polycystic ovaries diagnosed by ultrasound versus endocrine characteristics of PCOS. Fertil Steril 1997;67:452-8. 
Brasileiros De Cardiologia [serial on the Internet]. (2005, Apr), [cited July 26, 2018]; 84 Suppl 11-28.

26. CHEN, B. Y. Acupuncture normalizes dysfunction of hypothalamic-pituitary-ovarian axis. Acupuncture and Electro-Therapeutics, v. 22, p. 97-108, 1997. 